\title{
Administration of anti-vascular endothelial growth factor antibody following hepatectomy does not inhibit remnant liver regeneration or growth of remnant metastases
}

\author{
KAZUHIKO KASUYA $^{1}$, MINAKO SUZUKI ${ }^{1}$, YUICHI NAGAKAWA ${ }^{1}$, YOSHIAKI SUZUKI ${ }^{1}$, SATORU KIKUCHI ${ }^{1}$, \\ BUNSO KYO $^{1}$, TAKAAKI MATSUDO ${ }^{1}$, TAKAO ITOI ${ }^{2}$, AKIHIKO TSUCHIDA $^{1}$ and TATSUYA AOKI $^{1}$ \\ Departments of ${ }^{1}$ Digestive Surgery and ${ }^{2}$ Internal Medicine, Tokyo Medical University, Tokyo, Japan
}

Received September 28, 2011; Accepted December 2, 2011

DOI: $10.3892 /$ etm.2011.409

\begin{abstract}
In addition to the use of chemotherapeutic agents for the prevention of multiple liver metastases from colorectal cancer, the anti-vascular endothelial growth factor (VEGF) antibody, bevacizumab, is often used, and its effectiveness has been established. By contrast, it has been reported that the use of bevacizumab prior to or following surgery delays wound healing or liver regeneration. In this study, we investigated whether the administration of bevacizumab following hepatectomy inhibits remnant liver regeneration or the growth of remnant metastases. Mice were partially hepatectomized (31\% of the liver was removed), transplanted with the murine colorectal cancer cell line, CT26, in the remnant lobe, and intraperitoneally injected with bevacizumab $(4 \mathrm{mg} / \mathrm{kg})$ for a total of 6 times. Serum VEGF levels were measured on day 1 following surgery, and each lobe of the liver was weighed on day 14. Serum VEGF levels in nonhepatectomized, tumor-bearing mice exceeded those in their non-tumor-bearing counterparts; however, the administration of bevacizumab did not reduce the serum VEGF levels. The volume of the liver lobe of the hepatectomized, CT26-transplanted and non-CT26-transplanted mice was $1,349.6$ and $735.5 \mathrm{mg}$, respectively, indicating rapid growth of the CT26 transplant $(\mathrm{p}=0.023)$. The volume of the CT26-transplanted lobe of the bevacizumab-administered mice was $1,379.0 \mathrm{mg}$, which was not significantly different from that $(1,349.6 \mathrm{mg})$ of the nonbevacizumab-administered mice. The volume of the remnant lobe of the bevacizumab-administered mice was $1,051.0 \mathrm{mg}$, which did not significantly differ from that $(957.3 \mathrm{mg})$ of the non-bevacizumab-administered mice. The administration of bevacizumab following hepatectomy did not delay remnant liver regeneration, and did not suppress the growth of metastases in the remnant lobes or remnant liver regeneration.
\end{abstract}

Correspondence to: Dr Kazuhiko Kasuya, Department of Digestive Surgery, Tokyo Medical University Hospital, 6-7-1 Nishishinjuku, Shinjukuku, Tokyo 167-0023, Japan

E-mail: kasuya-k@jcom.home.ne.jp

Key words: vascular endothelial growth factor, antibody, hepatectomy, regeneration

\section{Introduction}

Over the years, advances in surgical resection procedures, chemotherapeutic agents and molecular-targeted drugs have led to an increase in the number of patients undergoing hepatectomy following neoadjuvant chemotherapy (NAC) for multiple liver metastases from colorectal cancer. No recurrence is generally observed in the remnant liver following complete surgical tumor resection. However, recurrence occurs in the remnant liver in a number of patients, indicating incomplete cancer resection or the persistence of micrometastases not visualized by imaging studies (1). Furthermore, if the future remnant liver volume and hepatic functional reserve are insufficient for the resection of multiple liver metastases, two-stage hepatectomy for initially unresectable multiple colorectal hepatic metastases is occasionally performed, in which case the cancer is knowingly left behind (2). Such patients at high risk of remnant liver recurrence need to undergo chemotherapeutic therapy as adjuvant chemotherapy (AC) and molecular-targeted therapy in the early post-operative period, as the levels of tumor growth factors, such as the vascular endothelial growth factor (VEGF), basic fibroblast growth factor (bFGF) and hepatocyte growth factor (HGF), are higher in the liver tissue of patients with colorectal liver metastases than in that of healthy controls, and become even higher following hepatectomy, due to liver regeneration $(3,4)$. It is well-known that when chemotherapy or molecular-targeted therapy is withheld following hepatectomy, the residual tumor enlarges (5). VEGF plays a central role in angiogenesis, and the VEGF-neutralizing antibody, bevacizumab, is one of the most useful drugs in suppressing tumor growth. However, VEGF has also been shown to increase the production of growth factors, such as HGF, and induce bone marrow cells, including vascular endothelial precursor cells, at sites of injury. This means that VEGF is involved not only in angiogenesis but also in liver cell regeneration following hepatectomy (6). In other words, there is concern that the administration of bevacizumab in the early period following hepatectomy may negatively affect the hypertrophy of the remnant liver and recovery of the liver function. The purpose of this study was to evaluate the effect of bevacizumab on the regeneration of the remnant liver following hepatectomy and to investigate whether bevacizumab exhibits an antitumor effect 
on the residual tumor during the phase of liver regeneration, during which various growth factors are secreted.

\section{Materials and methods}

Cell line and assay. The murine colon cancer cell line, CT26, which is an $\mathrm{N}$-nitroso-N-methylurethane-induced undifferentiated adenocarcinoma of the colon, syngeneic with the BALB/c mouse was purchased from the American Type Culture Collection (Manassas, VA, USA) for the purposes of this study. It was grown in cell culture as monolayers in RPMI-1640 medium with $2 \mathrm{mM}$ L-glutamine (Gibco Life Technologies Japan Ltd., Tokyo, Japan) supplemented with $10 \%$ fetal calf serum (FCS; Sigma, St. Louis, MO, USA), $100 \mathrm{U} / \mathrm{ml}$ penicillin and $100 \mu \mathrm{g} / \mathrm{ml}$ streptomycin in a humidified atmosphere containing 5\% $\mathrm{CO}_{2}$. To evaluate the VEGF protein expression of each mouse, we used a Quantikine mouse VEGF immunoassay (R\&D Systems Inc., Minneapolis, MN). The fluorescence of VEGF and the protein expression of the cells were normalized to those of the quantity of these proteins at the known level.

Animals. Animals were maintained at the Animal Care and Use Facilities at Tokyo Medical University Hospital under specific pathogen-free conditions. All experiments were approved by the Animal Care and Ethics Committee of Tokyo Medical University. Female BALB/c mice (CLEA Japan Inc., Tokyo, Japan) with a body weight (BW) of 20-22 $\mathrm{g}$ were used. The animals were housed in single cages at a room temperature of $22-24^{\circ} \mathrm{C}$ and a relative humidity of $60-65 \%$ with a $12-\mathrm{h}$ light/dark cycle environment. For all the operative procedures, animals were anesthetized by intraperitoneal (i.p.) injection of $50 \mathrm{mg} / \mathrm{kg} \mathrm{BW}$ pentobarbital sodium. Mice were placed on heated pads and the transverse upper abdominal incision was used to expose all liver lobes. A medium-large hemostatic clip (Ligaclip MCA, Ethicon Endo-surgery, Inc., Cincinnati, OH, USA) was applied across the pedicle of the right anterior (RA) and the left anterior (LA) lobes. These lobes were cut distal to the allied clip. The RA and LA lobes, which were equivalent to a hepatectomy of approximately $33 \%$ of the total liver mass, were removed. The abdomen was irrigated with $10 \mathrm{ml}$ of sterile saline and closed (7). The cell suspension of CT26, which included $2 \times 10^{5}$ cell $/ 25 \mu \mathrm{l}$ phosphate-buffered saline (PBS), was mixed with $25 \mu 1$ in Matrigel matrix (BD Biosciences, San Jose, CA, USA) on ice in a 1:1 ratio. The mixture was implanted into the surface of the left posterior (LP) lobe by using a $27 \mathrm{G}$ needle under direct visualization. The puncture site was compressed using a cotton swab for $3 \mathrm{~min}$ to stop the bleeding. The negative control group was injected with Matrigel alone. Bevacizumab $(4 \mathrm{mg} / \mathrm{kg}$ ) or human IgG (Sigma) was administered by i.p. injection 3 times a week from the day prior to (-1 day) to 10 days after CT26 cell implantation (6 times in total) (Fig. 1). On day 14, each lobe [LP and remnant lobes (RLs)] was removed and weighed. The number of mice undergoing the experimental procedures was 4-10 in each group.

Hepatic regeneration and tumor progression. We reviewed the following points as regards hepatic regeneration and tumor progression: i) Changes in serum VEGF level, LP lobe volume and RL volume by undergoing or not undergoing hepatectomy in the cancer-bearing mice or the non-cancer-bearing mice;

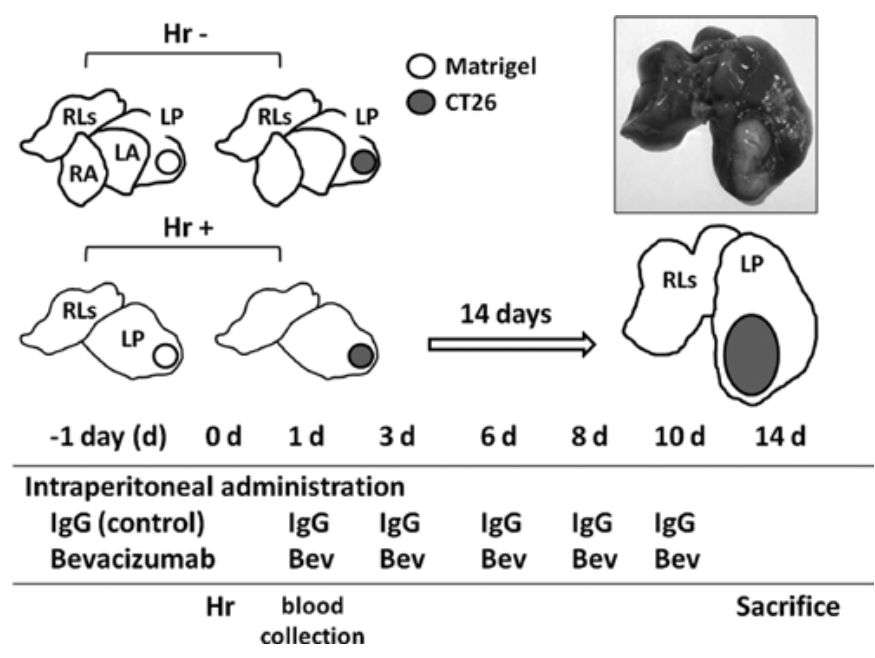

Figure 1. The uper left section shows the mouse liver consisting of 7 lobes (contribution of each lobe to the total liver weight): RA, right anterior (22\%); LA, left anterior (12\%); LP, left posterior (37\%); RLs, remnant lobes which consist of right posterior, right middle and two omental lobes (29\%). Hepatic resection group ( $\mathrm{Hr}+)$, mice undergoing the RA and the LA lobectomy; Hr-, mice without hepatic resection (Hr-). Open circle, matrigel injection; gray circle, CT26 injection. The upper right section shows the murine liver at 14 days after undergoing the RA and the LA lobectomy, and CT26 transplantation into the LP lobe. The lower section shows the schedule of administrations of bevacizumab (Bev) or $\mathrm{IgG}$ as the control, hepatic resection, blood collection and sacrifice.

ii) Changes in serum VEGF level, LP lobe volume, estimated tumor volume and the RL volume by undergoing or not undergoing hepatectomy, and by treating the cancer-bearing mice with or without bevacizumab. The estimated tumor volume was calculated as follows: The average volume of CT26 transplant LP lobe - the average volume of Matrigel transplant LP lobe.

Statistical analysis. Statistical analyses were performed using StatView software (Abacus Concepts Inc., Berkely, CA, USA). The serum VEGF level and the lobe volume were compared using the Mann-Whitney U test. A two-sided p-value of $<0.05$ was considered to indicate a statistically significant difference.

\section{Results}

Effect of hepatectomy in tumor-bearing and non-tumorbearing mice. The mean serum VEGF levels (1 day after laparotomy) of the non-hepatectomized, CT26-transplanted and non-CT26-transplanted mice (Matrigel-transplanted mice) were $113.4 \pm 61.6$ and $89.8 \pm 27.7 \mathrm{pg} / \mathrm{ml}$, respectively. There were no significant differences in these values between the groups [not significant (NS)]. The mean serum VEGF levels (1 day following hepatectomy) of the hepatectomized, CT26transplanted and non-CT26-transplanted mice were 29.6 \pm 11.4 and $19.3 \pm 7.7 \mathrm{pg} / \mathrm{ml}$, respectively (NS). The serum VEGF levels were significantly higher in the non-hepatectomized than in the hepatectomized mice ( $\mathrm{p}=0.010$ in the transplanted groups vs. $\mathrm{p}<0.05$ in the non-transplanted groups) (Fig. 2A). The mean volume of the LP lobe of the non-hepatectomized, CT26-transplanted and non-CT26-transplanted mice was $845.3 \pm 195.7$ and $606.2 \pm 41.3 \mathrm{mg}$, respectively (NS). The 

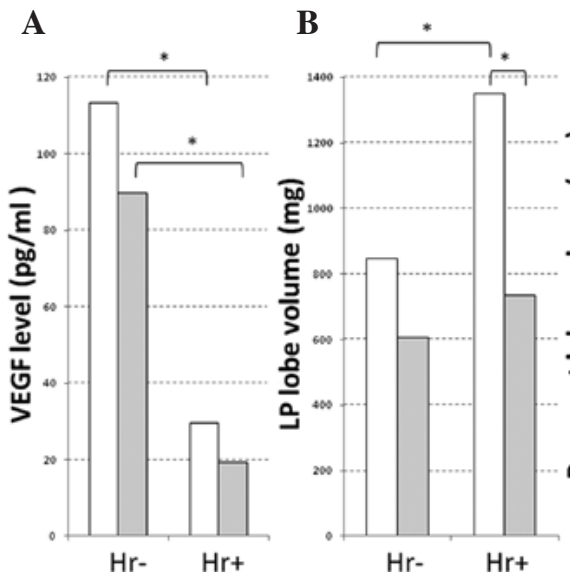

C

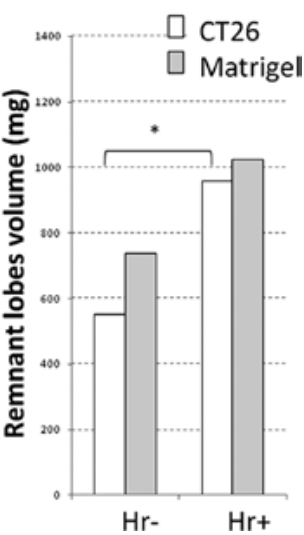

Figure 2. Comparison of (A) serum VEGF level, (B) left posterior (LP) lobe volume and $(\mathrm{C})$ remnant lobe $(\mathrm{RL})$ volume between tumor-bearing and non-tumor-bearing mice. The serum VEGF levels were significantly higher in the non-hepatectomized ( $\mathrm{Hr}-)$ than in the hepatectomized mice ( $\mathrm{Hr}+)$. The volume of the LP lobe was greatly enlarged in the hepatectomized and CT26-transplanted mice. The volume of the RLs was also enlarged significantly in the hepatectomized and CT26-transplanted mice. VEGF, vascular endothelial growth factor." $\mathrm{p}<0.05$.

increase in the volume of the LP lobe in the CT26-transplanted mice reflected the volume of the CT26 cells themselves. The mean volume of the LP lobe of the hepatectomized, CT26-transplanted and non-CT26-transplanted mice was $1,349 \pm 366.5$ and $735.5 \pm 62.3 \mathrm{mg}$, respectively, with a significant difference $(\mathrm{p}=0.023)$. In addition, a significant difference in the mean volume of the LB lobe was noted between the CT26 cell-transplanted, hepatectomized and non-hepatectomized mice; however, in the non-transplanted mice, the volume of the LP lobe increased slightly following hepatectomy, with no significant difference (Fig. 2B). The mean volume of the residual lobes (RLs) of the non-hepatectomized, CT26-transplanted and non-CT26-transplanted mice was 551.9 \pm 71.0 and $736.6 \pm 101.4 \mathrm{mg}$, respectively, and that of the RLs of the hepatectomized, CT26-transplanted or non-CT26-transplanted mice was $957.3 \pm 195.6$ and $1,025.0 \pm 163.2 \mathrm{mg}$, respectively, with no significant difference associated with CT26 cell transplantation. By contrast, the mean volume of the RLs was significantly larger in the hepatectomized, CT26-transplanted mice than in their non-hepatectomized counterparts $(\mathrm{p}=0.016)$, but not significantly greater than in their non-CT26-transplanted counterparts ( $\mathrm{p}=0.126$ ) (Fig. 2C), indicating that regeneration of the remnant liver occurs regardless of the presence or absence of cancer.

Effect of bevacizumab administration in CT26-transplanted mice. The mean serum VEGF level of the non-hepatectomized, bevacizumab-administered and non-bevacizumab-administered mice was 75.2 and $113.4 \mathrm{ng} / \mathrm{ml}$, respectively, and that of their hepatectomized counterparts was 63.8 and $29.9 \mathrm{ng} / \mathrm{ml}$, respectively (Fig. 3A; NS). The mean LP volume of the non-hepatectomized bevacizumab-administered and non-bevacizumab-administered mice was 871.4 and $845.3 \mathrm{mg}$, respectively, and that of their hepatectomized mice was $1,379.0$ and 1,349.0 mg, respectively (Fig. 3B; NS). The estimated tumor volume in the non-hepatectomized, bevacizumab-administered and non-bevacizumab-adminis-
A

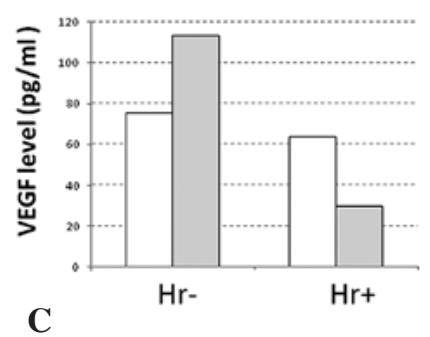

C

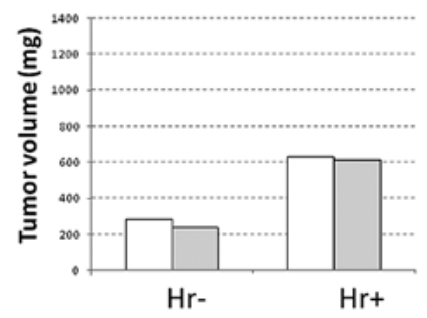

B
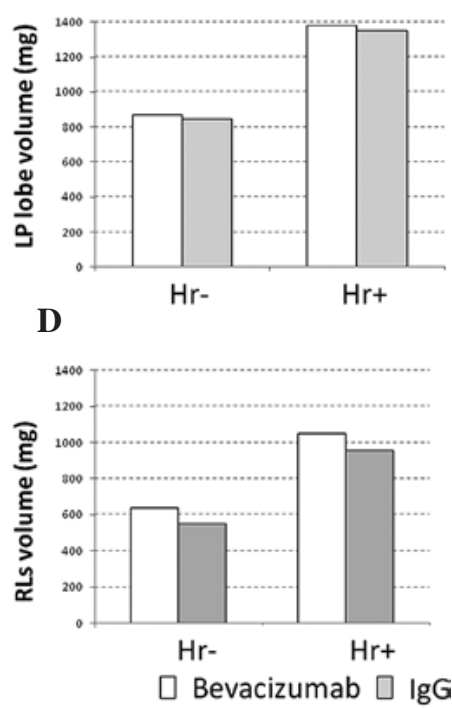

Figure 3. Comparison of (A) serum VEGF level, (B) left posterior lobe volume, (C) tumor volume and (D) remnant lobes volume between tumor-bearing and non-tumor-bearing mice with and without bevacizumab administration in CT26-transplanted mice. In the elements mentioned above, there was no significant difference between bevacizumab administration or no bevacizumab administration. VEGF, vascular endothelial growth factor; Hr-, non-hepatectomized mice; $\mathrm{Hr}+$, hepatectomized mice.

tered mice was 284.6 and $239.2 \mathrm{mg}$, respectively, and that of their hepatectomized counterparts was 628.0 and $613.4 \mathrm{mg}$, respectively (Fig. 3C; NS). The mean volume of the RLs in the non-hepatectomized, bevacizumab-administered and non-bevacizumab-administered mice was 637.3 and $551.9 \mathrm{mg}$, respectively, and that of their hepatectomized counterparts was $1,051.0$ and $957.3 \mathrm{mg}$, respectively (Fig. 3D; NS).

\section{Discussion}

Marked advances in chemotherapy and hepatic surgical procedures for liver metastases from colorectal cancer have led to an increase in the number of patients who have undergone hepatectomy for multiple liver metastases from colorectal cancer, which were previously deemed unresectable $(1,2)$. A number of these patients have undergone NAC. At this point, it is not clear whether NAC is capable of suppressing recurrence following hepatectomy. NAC reportedly has advantages in that the frequency of complete surgical tumor resection (R0) increases if chemotherapy reduces the tumor size, and that the serum levels of tumor growth factors, including VEGF and HGF, are higher in tumor-bearing patients than in healthy controls $(3,4)$. NAC is expected to reduce the levels of tumor growth factors in tumor-bearing patients. In the present study, the serum VEGF level was also higher in the CT26-transplanted than in the Matrigel-transplanted mice. In order to investigate what changes would occur in the levels of various growth factors following hepatectomy in tumorbearing mice with high levels of these growth factors, using a mouse hepatectomy model, Meredith et al (8) measured HGF and bFGF levels following hepatectomy, and demonstrated that their levels increased $24 \mathrm{~h}$ later, stating that HGF and bFGF were involved in liver regeneration. Taniguchi et al (9) 
reported the overexpression of VEGF in hepatocytes in the periportal areas of the rat, which are considered to be the primary site of hepatocyte regeneration, at 48-72 $\mathrm{h}$ following hepatectomy. Yamamoto et al (10) also reported an increase in the VEGF level in the portal blood following rat hepatectomy. These two reports suggested that VEGF, which was secreted by periportal hepatocytes, was closely involved in liver regeneration. In the present study, the serum VEGF level in the peripheral blood was lower in the hepatectomized than in the non-hepatectomized mice, suggesting that VEGF is expressed in hepatocytes in the remnant liver, that is, the regenerating liver itself. In the above-cited report (10), the VEGF level changed less in the peripheral blood than in the portal vein blood. These two reports suggest that VEGF is involved in liver regeneration, but that this involvement only occurs at sites of liver regeneration, and is not directly correlated with peripheral blood VEGF levels. In order to investigate the effect of tumor growth factors, including VEGF, on tumors in the remnant liver, Meredith et al (8) transplanted tumors in the remnant liver in a mouse model of liver resection, resulting in significantly enhanced tumor growth in hepatectomized mice in comparison with non-hepatectomized mice, which was ascribed to increased expression of HGF and bFGF. Similar results were observed in the present study, where the tumor enlarged rapidly following hepatectomy. However, we could not demonstrate the direct involvement of peripheral blood VEGF in tumor growth, or the antitumor effect of anti-VEGF antibodies. Although the key tumor growth factor has not been identified, we occasionally encounter such a phenomenon (i.e., rapid growth of tumors in the remnant liver) in clinical practice, and, naturally, we realize again the importance of not leaving any presence of the tumor in the remnant liver.

Advances in chemotherapy for liver metastases from colorectal cancer have led to a reduction in the size of liver metastases and improved the rate of hepatectomy. However, the rate of recurrence following hepatectomy is almost $80 \%(1,11)$, since micrometastases and tumor scars, which were deemed a complete response (CR) on imaging studies following chemotherapy, remain in the remnant liver. Indeed, Benoist et al (12) reported that no viable cancer cells were observed histopathologically in approximately $60 \%$ of lesions from patients shown to have CR on imaging studies. This indicates that some patients must continue to receive chemotherapy following surgery. Bevacizumab is one of the most useful drugs for the treatment of liver metastases from colorectal cancer. In addition, studies have reported the effect of bevacizumab on liver regeneration. Bockhorn et al (13) showed that the administration of VEGF during liver regeneration following partial hepatectomy promoted liver regeneration in the treatment group compared to the control group, and that the administration of anti-VEGF antibody delayed liver regeneration. In the present study, the administration of bevacizumab in the hepatectomized, tumorbearing mice did not delay remnant liver regeneration, which did not confirm the results of Bockhorn et al (13). However, at the same time, they reported that VEGF did not improve liver regeneration and survival following $90 \%$ subtotal liver resection (14). Therefore, it appears that the effects of VEGF and anti-VEGF antibody alone are insufficient to explain the mechanism of liver regeneration. The present study showed that the volume of the non-tumor region of the CT26-transplanted LP lobe increased following hepatectomy, but not so rapidly as the tumor, that the volume of the RLs following hepatectomy increased significantly regardless of the presence or absence of cancer, and that the administration of anti-VEGF antibody did not suppress the enlargement of the remnant liver.

In conclusion, the administration of anti-VEGF antibody in tumor-bearing mice following hepatectomy does not delay remnant liver regeneration. We found that the tumor in the remnant liver following hepatectomy grew rapidly, and the administration of anti-VEGF did not suppress its growth. Thus, the activity of anti-VEGF antibody was assumed to be negligible compared with the major changes caused by hepatectomy.

\section{Acknowledgements}

We thank Mr. Hiroaki Tanaka and Hiroshi Ohta, university students who belong to the Department of Clinical Pharmacy of Tokyo University of Pharmacy and Life Sciences for their valuable technical assistance.

\section{References}

1. Adam R, Delvart V, Pascal G, et al: Rescue surgery for unresectable colorectal liver metastases downstaged by chemotherapy: a model to predict long-term survival. Ann Surg 240: 644-657, 2004.

2. Adam R, Miller R, Pitombo M, Wicherts DA, De Haas RJ, Bitsakou G and Aloia T: Two-stage hepatectomy approach for initially unresectable colorectal hepatic metastases. Surg Oncol Clin N Am 16: 525-536, 2007.

3. Fukuura T, Miki C, Inoue T, Matsumoto K and Suzuki H: Serum hepatocyte growth factor as an index of disease status of patients with colorectal carcinoma. Br J Cancer 78: 454-459, 1998.

4. Yoon SS, Kim SH, Gonen M, et al: Profile of plasma angiogenic factors before and after hepatectomy for colorectal cancer liver metastases. Ann Surg Oncol 13: 353-362, 2006

5. Mentha G, Terraz S, Morel P, et al: Dangerous halo after neoadjuvant chemotherapy and two-step hepatectomy for colorectal liver metastases. Br J Surg 96: 95-103, 2009.

6. Folkman J and Shing Y: Angiogenesis. J Biol Chem 267: 10931-10934, 1992.

7. Greene AK and Puder M: Partial hepatectomy in the mouse: technique and perioperative management. J Invest Surg 16: 99-102, 2003.

8. Meredith K, Haemmerich D, Qi C and Mahvi D: Hepatic resection but not radiofrequency ablation results in tumor growth and increased growth factor expression. Ann Surg 245: 771-776, 2007.

9. Taniguchi E, Sakisaka S, Matsuo K, Tanikawa K and Sata M: Expression and role of vascular endothelial growth factor in liver regeneration after partial hepatectomy in rats. J Histochem Cytochem 49: 121-130, 2001.

10. Yamamoto C, Yagi S, Hori T, Iida T, Taniguchi K, Isaji S and Uemoto S: Significance of portal venous VEGF during liver regeneration after hepatectomy. J Surg Res 159: 37-43, 2010.

11. Alberts SR, Horvath WL, Sternfeld WC, et al: Oxaliplatin, fluorouracil, and leucovorin for patients with unresectable liver-only metastases from colorectal cancer: a North Central Cancer Treatment Group phase II study. J Clin Oncol 23: 9243-9249, 2005.

12. Benoist S, Brouquet A, Penna C, et al: Complete response of colorectal liver metastases after chemotherapy: does it mean cure? J Clin Oncol 24: 3939-3945, 2006.

13. Bockhorn M, Goralski M, Prokofiev D, et al: VEGF is important for early liver regeneration after partial hepatectomy. J Surg Res 138: 291-299, 2007.

14. Bockhorn M, Schöllmann S, Opitz B, et al: Vascular endothelial growth factor does not improve liver regeneration and survival after 90\% subtotal liver resection. Hepatol Res 37: 353-359, 2007. 